\title{
Theory of collective firing induced by noise or diversity in excitable media
}

\author{
C. J. Tessone, A. Scirè, R. Toral, and P. Colet \\ Institut Mediterrani d'Estudis Avançats, IMEDEA (CSIC-UIB), Campus Universitat Illes Balears, E-07122 Palma de Mallorca, Spain
}

(Received 20 June 2006; published 9 January 2007)

\begin{abstract}
Large variety of physical, chemical, and biological systems show excitable behavior, characterized by a nonlinear response under external perturbations: only perturbations exceeding a threshold induce a full system response (firing). It has been reported that in coupled excitable identical systems noise may induce the simultaneous firing of a macroscopic fraction of units. However, a comprehensive understanding of the role of noise and that of natural diversity present in realistic systems is still lacking. Here we develop a theory for the emergence of collective firings in nonidentical excitable systems subject to noise. Three different dynamical regimes arise: subthreshold motion, where all elements remain confined near the fixed point; coherent pulsations, where a macroscopic fraction fire simultaneously; and incoherent pulsations, where units fire in a disordered fashion. We also show that the mechanism for collective firing is generic: it arises from degradation of entrainment originated either by noise or by diversity.
\end{abstract}

DOI: 10.1103/PhysRevE.75.016203

PACS number(s): 05.45.Xt, 05.40.-a, 02.50.-r

\section{INTRODUCTION}

Excitable behavior appears in a large variety of physical, chemical, and biological systems [1,2]. This behavior occurs typically for parameter values close to an oscillation bifurcation, and is characterized by a nonlinear response to perturbations of a stationary state: while small perturbations induce a smooth return to the fixed point, perturbations exceeding a given threshold induce a return through a large phase space excursion (firing), largely independent of the magnitude of the perturbation. Furthermore, after one firing the system cannot be excited again within a refractory period of time. In many situations of interest, the firings are induced by random perturbations or noise [3].

In coupled excitable systems, macroscopic firing (a significatively large fraction of the units fire simultaneously) excited by noise has been observed in chemical excitable media [4,5], neuron dynamics [6], and electronic systems [7], and it has been described through several theoretical approaches [8-10]. This synchronized firing can be considered as a constructive effect induced by the noise. Other examples in which noise actually helps to obtain a more ordered behavior are stochastic resonance [11], stochastic coherence (or coherence resonance) [12], and noise-induced phase transitions [13].

Diversity, the fact that not all units are identical, is an important ingredient in realistic modeling of coupled systems. Ensembles of coupled oscillators with diversity have been paradigmized [14] and largely studied $[15,16]$, with the result that synchronized behavior can appear once the disorder induced by the diversity is overcome by the entraining effect of the coupling. In particular, $[17,18]$ study analytically the transition to desynchronization in an active rotator model in the oscillatory, high natural frequency limit.

Also, it has been shown that in a purely deterministic excitable system diversity may induce collective firing [19] if a fraction of the elements are above the oscillatory bifurcation. In view of these examples, it might be expected that diversity and noise can play a similar role.

In this work, we develop an analytical understanding for the emergence of collective firing in coupled excitable sys- tems in presence of disorder, either induced by noise or diversity. We show that three different dynamical regimes are possible: subthreshold motion, where all elements remain confined near the fixed point; coherent pulsations, where a macroscopic fraction fire simultaneously; and incoherent pulsations, where units fire in a disordered fashion. Remarkably, the coherent behavior appears through a genuine phase transition when the noise intensity, the coupling or the diversity cross a critical value. A second phase transition to the disordered (incoherent) phase is recovered for large enough noise intensity or diversity, or small enough coupling. The mechanism for collective firing is the degradation of entrainment which can be originated either by noise or diversity. This is generic and opens a new scenario for experimental observations.

This paper is organized as follows: in the next section we present the model and introduce a general theoretical treatment to understand this problem. In Sec. III we compare the numerical simulations with the theory. Finally, in Sec. IV the conclusions are drawn.

\section{MODEL AND THEORY}

We consider as a prototypical model an ensemble of globally coupled active-rotators $\phi_{j}(t), j=1, \ldots, N$, whose dynamics is given by [20]

$$
\dot{\phi}_{j}=\omega_{j}-\sin \phi_{j}+\frac{C}{N} \sum_{k=1}^{N} \sin \left(\phi_{k}-\phi_{j}\right)+\sqrt{D} \xi_{j} .
$$

The natural frequencies $\omega_{j}$ are distributed according to a probability density function $g\left(\omega_{j}\right)$, with mean value $\omega$ and variance $\sigma^{2}$. Notice that $\omega_{j}<1$ (respectively, $\omega_{j}>1$ ) corresponds to an excitable (respectively, oscillatory [21]) behavior of the solitary rotator $j$. Throughout the paper we consider the case $\omega<1 . D$ is the intensity of the Gaussian noises $\xi_{j}$ of zero mean and correlations $\left\langle\xi_{j}(t) \xi_{k}(t)\right\rangle=\delta\left(t-t^{\prime}\right) \delta_{j k}$, and $C$ is the coupling intensity.

To characterize collective behavior we use the timedependent global amplitude, $\rho(t)$, and phase, $\Psi(t)[14,22]$, 


$$
\rho(t) e^{i \Psi(t)}=\frac{1}{N} \sum_{k=1}^{N} e^{i \phi_{k}(t)} .
$$

The Kuramoto order parameter $\rho \equiv\langle\rho(t)\rangle$, where \langle\rangle denotes the time average, is known to be a good measure of collective synchronization in coupled oscillators systems, i.e., $\rho$ $=1$ when oscillators synchronize $\phi_{j}(t)=\phi_{k}(t), \forall j, k$, and $\rho$ $\rightarrow 0$ for desynchronized behavior. In the excitable region there are two different regimes that could give raise to a value $\rho=1$. The first regime is a dynamical one in which the units fire pulses synchronously; this situation corresponds to full synchronization. The other regime is a static one, in which all the units rest in the stable fixed point. To discriminate between this static entrainment from the dynamic entrainment of excitable systems when all units fire synchronously, we use the order parameter introduced by Shinomoto and Kuramoto [22]

$$
\zeta=\left\langle\left|\rho(t) e^{i \Psi(t)}-\left\langle\rho(t) e^{i \Psi(t)}\right\rangle\right|\right\rangle,
$$

which differs from zero only in the case of synchronous firing. Finally, a measure for the activity of the units, widely used in problems of stochastic transport in nonsymmetric potentials is the current

$$
J=\frac{1}{N} \sum_{k=1}^{N}\left\langle\dot{\phi}_{k}(t)\right\rangle
$$

A nonzero current $J$ describes a situation in which the systems are firing (not necessarily synchronized).

We now provide an analytical theory to understand the behavior of $\rho, \zeta$, and $J$ as a function of the control parameters, $C, D$, and $\sigma$. The theory proceeds in three steps. First, under the assumption of entrainment, we derive a dynamical equation for the global phase $\Psi$, depending on the value of the Kuramoto parameter $\rho$. Second, using the solution of that equation, we obtain expressions for $\zeta$ and $J$ which depend on $\rho$. Finally, we calculate self-consistently the value of $\rho$.

Averaging Eq. (1) over the whole ensemble and using the definition of global amplitude and phase of Eq. (2) we have

$$
\frac{1}{N} \sum_{k=1}^{N} \dot{\phi}_{k}=\omega-\rho(t) \sin \Psi(t)+\sqrt{\frac{D}{N}} \xi(t),
$$

where $\xi(t)$ is a Gaussian noise of zero mean and correlations $\left\langle\xi(t) \xi\left(t^{\prime}\right)\right\rangle=\delta\left(t-t^{\prime}\right)$. Taking the time derivative of Eq. (2) and introducing $\delta_{j}(t)=\phi_{j}(t)-\Psi(t)$, we obtain

$$
\dot{\rho}(t)+i \rho(t) \dot{\Psi}(t)=\frac{i}{N} \sum_{k=1}^{N} \dot{\phi}_{k} e^{i \delta_{k}(t)} .
$$

We consider now that the rotators are synchronized in the sense that $\delta_{j}(t) \ll 1$ and substitute the expansion $e^{i \delta_{k}}=1+i \delta_{k}$ $+O\left(\delta_{k}^{2}\right)$ in the previous expression. Equating real and imaginary parts, we obtain

$$
\rho(t) \dot{\Psi}(t)=\frac{1}{N} \sum_{k=1}^{N} \dot{\phi}_{k}+O\left(\delta_{k}^{2}\right) .
$$

The definition of $\delta_{i}$ leads to $\rho(t)=N^{-1} \Sigma_{k} e^{i \delta_{k}}$. Hence $\dot{\rho}(t)$ $=O\left(\delta_{k}^{2}\right)$ and, consistently with the order of the approximation, we can replace in Eq. (7) the time dependent $\rho(t)$ by the constant value $\rho$. Therefore, Eq. (5) can be approximated by

$$
\rho \dot{\Psi}(t)=\omega-\rho \sin \Psi(t)+\sqrt{D / N} \xi(t),
$$

which in the limit $N \rightarrow \infty$, reduces to

$$
\dot{\Psi}(t)=\frac{\omega}{\rho}-\sin \Psi(t)
$$

It is remarkable that the global phase obeys the same dynamics than the individual units but with a natural frequency scaled with $\rho$, the Kuramoto parameter measuring the entrainment degree. Therefore, a decrease in the entrainment lowers the collective threshold from $\omega=1$ to $\omega=\rho$ and the system can start firing synchronously. The effect can be understood as a broadening of the distribution of the phases $\phi$, so that a fraction of the rotators crosses over the threshold and, if the coupling is large enough, they pull a macroscopic fraction of the oscillators. Thus degradation of the entrainment has the paradoxical effect of increasing the coherent firing. It is essential to realize that Eq. (9) depends only on the value of $\rho$ and not in the specific way the degradation of $\rho$ is achieved, so that similar effects can be achieved either increasing the noise, either decreasing the coupling, or increasing the diversity in the natural frequencies; a significantly insightful result not previously understood nor discussed.

We now turn our attention to the computation of the probability distribution $P(\Psi, \rho)$. As shown previously, $\rho$ is constant for small $\delta_{i}$, and then the probability density associated to the macroscopic equation (8), is

$$
P(\Psi ; \rho, \omega)=Z^{-1} e^{-2 N U(\Psi ; \rho, \omega) / D} \int_{0}^{2 \pi} d \Psi^{\prime} e^{2 N U\left(\Psi^{\prime}+\Psi ; \rho, \omega\right) / D}
$$

where $Z$ is a normalizing constant. The associated potential is given by

$$
U(\Psi ; \rho, \omega)=-\frac{\omega}{\rho} \Psi-\cos (\Psi)
$$

In the thermodynamic limit, the expression in Eq. (8) is deterministic, and then Eq. (9) gets simplified. Then, the distribution function which is proportional to $\sim 1 / \dot{\Psi}(t)$, is given by 


$$
P(\Psi ; \rho)=\left\{\begin{array}{l}
\frac{1}{2 \pi} \sqrt{\omega^{2}-\rho^{2}} /(\omega-\rho \sin \Psi) \text { for } \rho<\omega, \\
\delta[\Psi-\arcsin (\omega / \rho)] \text { for } \rho \geq \omega .
\end{array}\right.
$$

The case $\rho>\omega$, corresponds to the case in which the global phase dynamics has a fixed point, and then the distribution becomes a delta function.

Another interesting result of our approach, is that it allows us to express $\zeta$ and $J$ as a function of the Kuramoto order parameter $\rho$. In the case $\rho<\omega$, the solution of (9) is given by [23]

$$
\omega-\rho \sin \Psi(t)=\frac{\omega^{2}-\rho^{2}}{\omega-\rho \cos \Omega t},
$$

where $\Omega=\sqrt{(\omega / \rho)^{2}-1}$ is the frequency of the global phase oscillations. The current is obtained from Eq. (5), $J=\omega$ $-\langle\rho \sin (\Psi)\rangle$. Time averages are computed over a period $T=2 \pi / \Omega$ using Eq. (13),

$$
J=\frac{\omega^{2}-\rho^{2}}{T} \int_{0}^{T} \frac{d t}{\omega-\rho \cos \Omega t}=\sqrt{\omega^{2}-\rho^{2}} .
$$

For $\rho>\omega, J=0$.

Approximating $\rho(t)$ by a constant value, the ShinomotoKuramoto parameter $\zeta \cong \rho\left\langle\left|e^{\mathrm{i} \Psi(t)}-\left\langle e^{\mathrm{i} \Psi(t)}\right\rangle\right|\right\rangle$ can be computed performing again the time averages over a period $T$ using Eq. (13),

$$
\zeta=\frac{2}{\pi} \sqrt{2\left(\omega-\sqrt{\omega^{2}-\rho^{2}}\right)(\omega+\rho)} K\left(\frac{2 \rho}{\rho-\omega}\right),
$$

where $K(m)$ is the complete elliptic integral of the first kind [24]. If $\rho>\omega$ we get $\zeta=0$.

As a final step, we derive a equation for $\rho$ using a selfconsistent, Weiss-type, mean-field approximation, which assumes constant values for the global magnitudes and then averages over their probability distribution $[13,14]$. For our particular case, we start by rewriting Eq. (1) as

$$
\dot{\phi}_{i}(t)=-\frac{d V\left(\phi_{i} ; \Psi, \rho, \omega_{i}\right)}{d \phi_{i}}+\sqrt{D} \xi_{i}(t),
$$

where we have defined the potential

$$
V(\phi ; \Psi, \rho, \omega)=-\omega \phi-\cos (\phi)-C \rho \cos (\Psi-\phi) .
$$

Note that the coupling appears only through the global parameters $\rho$ and $\Psi$. For fixed $\rho$ and $\Psi$, the stationary probability distribution function reads [25]

$$
P_{\mathrm{st}}(\phi ; \Psi, \rho, \omega)=Z^{-1} e^{-2 V(\phi) / D} \int_{0}^{2 \pi} d \phi^{\prime} e^{2 V\left(\phi^{\prime}+\phi\right) / D},
$$

where $Z$ is a normalizing constant. From its definition, we have $\rho=(1 / N) \sum_{k=1}^{N}\left\langle\cos \left(\phi_{k}-\Psi\right)\right\rangle$, and we obtain
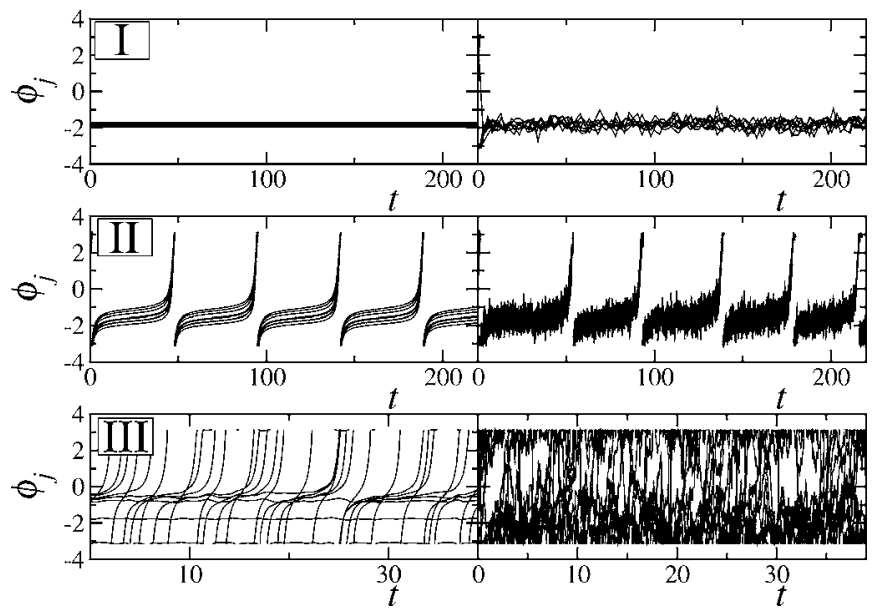

FIG. 1. Dynamical trajectories for 10 typical units in a system of $N=400$ with $\omega=0.95$ and $C=4$. The right-hand column depicts the results without noise $D=0$, and different diversities: $\sigma=0.0$ (top, regime I, no firing), $\sigma=1.6$ (middle, regime II, synchronized firing) and $\sigma=3.0$ (bottom, regime III, desynchronized firing). While the left-hand column shows the dynamical regimes emerging under the presence of noise (and no diversity): $D=0.4$ (top, regime I, no firing), $D=1.0$ (middle, regime II, synchronized firing) and $D=5.0$ (bottom, regime III, desynchronized firing).

$$
\begin{aligned}
\rho= & \int d \omega g(\omega) \int_{0}^{2 \pi} d \Psi P(\Psi ; \rho) \int_{0}^{2 \pi} d \phi P_{\mathrm{st}}(\phi ; \Psi, \rho, \omega) \\
& \times \cos (\phi-\Psi),
\end{aligned}
$$

where we have performed a triple average: with respect to the distribution (18), with respect to the distribution $g(\omega)$ of natural frequencies and with respect to the distribution $P(\Psi ; \rho)$ of the global phase which is inversely proportional to the instantaneous velocity given by Eq. (10). The selfconsistent equation (19) for $\rho$ needs to be solved numerically.

\section{NUMERICAL RESULTS AND DISCUSSION}

In the following, we discuss the theoretical results and compare them with the numerical results obtained from a numerical integration of Eq. (1). Typical trajectories showing the three dynamical regimes are displayed in Fig. 1 while the corresponding order parameters are plotted in Fig. 2. Figure 2(a) a shows $\rho, \zeta$, and $J$ as functions of the noise intensity $D$ in absence of diversity. The solid lines correspond to the theoretical results while symbols show the numerical results for different system sizes. In this figure, we can observe the three aforementioned behaviors: For small noise intensity (regime I) each rotator fluctuates around its fixed point. Although for uncoupled rotators noise would eventually excite some spontaneous random firings, the coupling of a large number of units suppresses these individual firings. The Kuramoto parameter $\rho$ remains close to 1 and the deviations from unity are due to the small dispersion induced by noise. Region I is, in fact, characterized by $\rho>\omega$ for which our theory predicts that the Shinomoto-Kuramoto parameter $\zeta$ 
(a)

(b)

(c)

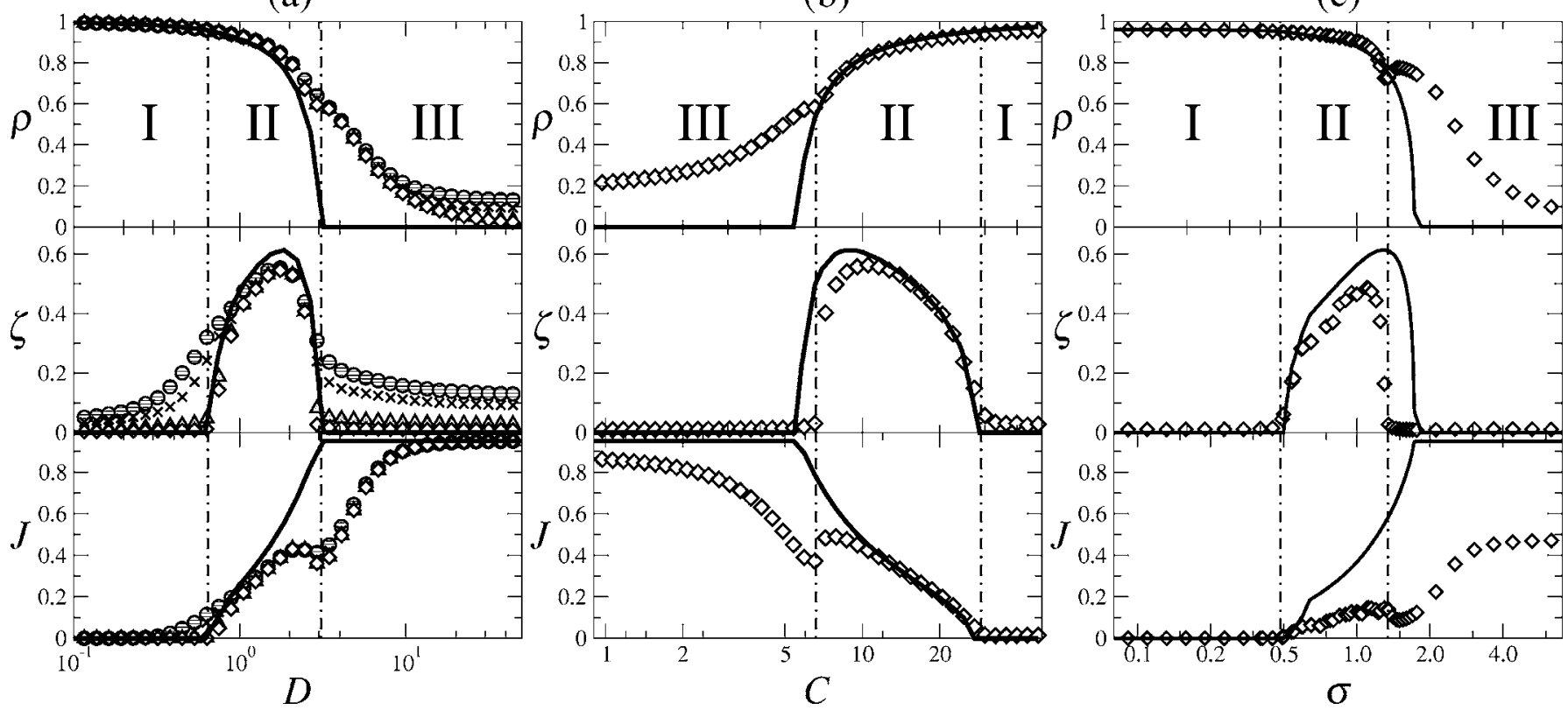

FIG. 2. Symbols represent $\rho, \zeta$, and $J$ as obtained numerically from Eq. (1). Solid lines are the theoretical results. Panel (a) shows the variation with respect to the noise intensity $D$ in absence of diversity, $\sigma=0$, for frequency $\omega=0.95$, coupling $C=4$, and different system sizes: $N=50(\circ), N=10^{2}(\times), N=10^{3}(\triangle), N=10^{4}(\diamond)$. Panel (b) displays the same results as a function of $C$ for $D=1.0$. Panel (c) shows the variation with respect to diversity $\sigma$ for $D=0.3, C=4$ and $g\left(\omega_{j}\right)$ being a uniform distribution. In all cases there are three regimes: (I) no firing, (II) synchronized firing, and (III) desynchronized firing.

and the current $J$ vanish, so reflecting the nonexistence of collective movement. In this region, the numerical results for $\rho, \zeta$, and $J$ are in excellent agreement with the theoretical predictions.

Our theory predicts that a transition to a dynamical state characterized by synchronized firing behavior (regime II) takes place when $\rho=\omega$ again, in very good agreement with the numerical results. This transition is clearly signaled by nonvanishing values of $\zeta$ and $J$. The prediction of $\rho$ is good for a large part of region II (up to values of $\rho=0.7$ ). Later it underestimates its value.

For very large noise intensity, the rotators desynchronize while keeping a nonzero current value (regime III). Hence, the synchronized activity, as measured by $\zeta$ goes though a maximum as noise amplitude increases. Our theory predicts that the transition between regions II and III occurs for $\rho$ $=0$ where $\zeta=0$ and the current takes the maximum possible value $J=\omega$. The limit value of $J$ for very large disorder can be understood by inspection of Eq. (5), due to the fact that in this limit $\rho$ vanishes, and that the time average of the noise term is zero. The transition from II to III corresponds to the expected disordering role of diversity (or noise) and it is the analogous of similar transitions in oscillator models, such as Kuramoto's: an increase of disorder desynchronizes the system. In this case, this transition is signaled by $\zeta=0$.

Surprisingly, since the small values of $\rho$ in this transition are beyond the assumptions of the theory, the location of the second transition is also well predicted. Moreover, the whole shape of the Shinomoto-Kuramoto parameter $\zeta$ is well reproduced over the whole range. The maximum of Eq. (15) occurs for $\rho \approx 0.821 \omega$, which is well confirmed by the numerical results. The theoretically predicted current $J$ fits the numerical values in the same range than $\rho$. Note, however, the numerical simulations show a local maximum for the current $J$ which indicates a local increasing in the total transport due to the coherent dynamics in the regime II. This local maximum is not present in the theoretical approximation.

Some of these states were already described by Kuramoto and Shinomoto [22]. By looking at the probability distribution of $\phi_{i}$, these authors identify two regions in parameter space: the time-periodic regime $(P)$ and the stationary regime $(S)$. Region $P$ corresponds to our regime II where the order parameter $\zeta$ is different from zero and there is collective motion of the oscillators. Our findings allow us to split region $S$ of these authors into our distinct regions I and III: while region I is a fluctuating regime around the steady state, region III has a high activity as characterized by a nonzero current $J$. In $[8,9]$, a semianalytical approach was used to analyze the existence of these three phases. However, an explanation of why these regimes appear, and the finding of general mechanisms that could trigger the collective behavior in these systems, was lacking.

Our results indicate that noise acts in two antagonistic ways: while a given noise intensity can excite the subthreshold units, forcing a synchronized firing, large amplitude noise deteriorates the synchronization properties of the ensemble. This scenario resembles the so-called noise induced phase transitions [13] in which a transition to an ordered ferromagneticlike state is induced by increasing the noise intensity; the order is destroyed again for large enough noise. Here, the transition is towards an organized collective motion of the active rotators.

The reverse scenario can be observed varying the coupling strength $C$, see Fig. 2(b). The Kuramoto parameter $\rho$ 
increases with $C$ (notice, however, the existence of a small bump in the numerical results), indicating that the degree of synchronization increases with coupling, as expected. A large coupling suppresses noise-induced firings, and the system is macroscopically at rest, regime I, as indicated by the vanishing of $\zeta$ and $J$. For weak coupling the noise induces desynchronized individual firings (regime III) characterized by a macroscopic current $J$ and again a zero value for $\zeta$. For intermediate values of the coupling (regime II) the interplay of noise and coupling leads to the largest degree of synchronized firing with a large value for $\zeta$.

Finally Fig. 2(c) shows $\rho, \zeta$, and $J$ as a function of the diversity $\sigma$. It is clear in the figure the existence of the same three regimes that were obtained by varying the noise intensity or the coupling. Altogether Fig. 2 clearly illustrates the fact that similar effects can be achieved increasing the noise, decreasing the coupling or increasing the diversity in the natural frequencies as theoretically predicted.

\section{CONCLUSIONS}

In summary, we have developed a theory for the emergence of collective firing in a paradigmatic ensemble of subthreshold excitable units, containing coupling and a source of disorder as generic ingredients. The collective behavior emerges as a phase transition whose underground mechanism is the degradation of entrainment originated by the competing effects of disorder and coupling. Paradoxically, this deg- radation results in establishing a lower effective threshold for collective firing, and thus inducing a somehow ordered state. Our theory clearly establishes that it does not matter the specific source of disorder, either noise or diversity will lead to similar results. This mechanism is not restricted to the model we considered, it will exist in any physical, chemical or biological excitable system with the aforementioned basic generic ingredients. Our results are likely to be relevant also for nonglobally coupled systems, such as extended systems with local couplings and complex networks.

In extended systems the macroscopic fraction of units firing collectively are typically localized in a region of the space, leading to the propagation of an excited wave. Waves induced by parametric noise in chemical excitable systems had been reported [4,5]. It has been argued in [9] that the transition between I and II would be triggered by changes in the excitability of the neurons, if one assumes that noise intensity is a constant. In this paper we show a more plausible scenario by which biological systems can trigger such transition by adjusting the level of diversity among the neurons. We expect that our results will stimulate further experiments on systems with different sources of disorder.

\section{ACKNOWLEDGMENTS}

The authors acknowledge financial support by the Ministerio de Educación y Ciencia (Spain), FEDER Contract Nos. FIS2004-5073, FIS2004-953, BFM2001-0341 and the EU NoE BioSim (LSHB-CT-2004-005137).
[1] J. D. Murray, Mathematical Biology (Springer-Verlag, New York, 2002), Chap. 11.

[2] E. Meron, Phys. Rep. 218, 1 (1992).

[3] B. Lindner, J. García-Ojalvo, A. Neiman, and L. SchimanskyGeier, Phys. Rep. 392, 321 (2004).

[4] S. Kadar, J. Wang, and K. Showalter, Nature (London) 391, 770 (1998).

[5] S. Alonso, I. Sendiña-Nadal, V. Pérez-Muñuzuri, J. M. Sancho, and F. Sagués, Phys. Rev. Lett. 87, 078302 (2001).

[6] S. Tanabe and K. Pakdaman, Biol. Cybern. 85, 269 (2001).

[7] D. E. Potsnov, S. K. Han, T. G. Yim, and O. V. Sosnovtseva, Phys. Rev. E 59, R3791 (1999).

[8] M. A. Zaks, A. B. Neiman, S. Feistel, and L. SchimanskyGeier, Phys. Rev. E 68, 066206 (2003).

[9] C. Kurrer and K. Schulten, Phys. Rev. E 51, 6213 (1995).

[10] S. H. Park and S. Kim, Phys. Rev. E 53, 3425 (1996).

[11] L. Gammaitoni, P. Hänggi, P. Jung, and F. Marchesoni, Rev. Mod. Phys. 70, 223 (1998).

[12] A. S. Pikovsky and J. Kurths, Phys. Rev. Lett. 78, 775 (1997).

[13] C. van den Broeck, J. M. R. Parrondo, and R. Toral, Phys. Rev. Lett. 73, 3395 (1994).
[14] Y. Kuramoto, Chemical Oscillations, Waves, and Turbulence (Springer-Verlag, New York, 1984).

[15] S. Strogatz, Physica D 143, 1 (2000).

[16] S. Strogatz, Nonlinear Dynamics and Chaos (Perseus Books, Cambridge, 1994).

[17] J. A. Acebrón and L. L. Bonilla, Physica D 114, 296 (1998).

[18] J. A. Acebrón, L. L. Bonilla, C. J. Pérez Vicente, F. Ritort, and R. Spigler, Rev. Mod. Phys. 77, 137 (2005).

[19] J. H. E. Cartwright, Phys. Rev. E 62, 1149 (2000).

[20] Y. Kuramoto, in International Symposium on Mathematical Problems in Theoretical Physics, 1975, p. 39.

[21] In this case, note that actual frequency is $\sqrt{\omega^{2}-1}$.

[22] S. Shinomoto and Y. Kuramoto, Prog. Theor. Phys. 75, 1105 (1986).

[23] This form of the solution assumes a particular initial condition $\Psi(t=0)=-\pi / 2$, which is otherwise irrelevant when taking the time average.

[24] M. Abramowitz and I. A. Stegun, Handbook of Mathematical Functions (Dover, New York, 1964), Chap. 17, p. 589.

[25] C. W. Gardiner, Handbook of Stochastic Methods (SpringerVerlag, Berlin, 1985), Chap. 5, p. 125. 